\title{
14 PRODUCTION PLANNING AND CONTROL IN A VIRTUAL ENTERPRISE
}

\author{
L.M. Camarinha-Matos ${ }^{1 \bullet}$, V. Santos Silva ${ }^{2}$, R. J. Rabelo ${ }^{3}$ \\ ${ }^{1}$ New University of Lisbon, Portugal \\ ${ }^{2}$ CSIN Lda, Portugal \\ ${ }^{3}$ Federal University of Santa Catarina, Brazil
}

\begin{abstract}
The Production Planning and Control system (PPC) typically plays a major role among the legacy applications of an enterprise that joins an industrial virtual enterprise environment. The PPC is both the source and the destination of most of the information exchanged between partner enterprises. In order to support the operation in a virtual enterprise environment, typical legacy PPC systems have to be expanded and re-engineered. Some proposals and acquired experiences in this direction are discussed.
\end{abstract}

\section{INTRODUCTION}

\section{The role of a PPC}

The Production Planning and Control system (PPC) is one of the most important applications in an industrial company. Although there is not a total consensus about its boundaries, a typical PPC system includes several functions such as: (i) Industrial logistics management - involving orders flow management, product data management, sales forecasts handling, and actual requirements planning; (ii) Master production scheduling; (iii) Material requirements planning; (iv) Capacity requirements planning; (v) Production control and scheduling; (vi) Quality control and tracking; and (vii) Industrial costing.

When an enterprise participates in a manufacturing virtual enterprise (VE), its PPC is the main source and destination of the information exchanged between this enterprise and its VE partners. For instance when a company sends a purchasing order to another company, the PPC of the sender company is the source of information for that order whereas the PPC at the receiving company is the destination (consumer) of the order.

\footnotetext{
- Corresponding author address: Universidade Nova de Lisboa, Quinta da Torre, 2825 Monte Caparica, Portugal, tel. +351-1-2948517, fax +351-1-2941253, e-mail: cam@uninova.pt.
} 
Building a system to integrate all manufacturing and engineering support components has been the focus of attention of many research activities during several years and is outside of the scope of PRODNET II. Within this project, one of the goals was rather to investigate the extensions and re-engineering required by a typical legacy PPC system in order to support the enterprise's operation in a VE environment.

\section{The PPC in the PRODNET architecture}

Figure 1 shows the place of the PPC system in the PRODNET reference architecture. As it is illustrated, the PPC system is part of the so-called internal module that represents the various enterprise applications and legacy systems that run in the enterprise like a PPC or ERP system, a CAD system, other engineering tools, etc. The PRODNET Cooperation Layer (PCL) is the component responsible for supporting all interactions between a company and its VE partners. Therefore, the interactions between the PPC of a particular enterprise and its VE partners are done through the local PCLs of the involved companies.
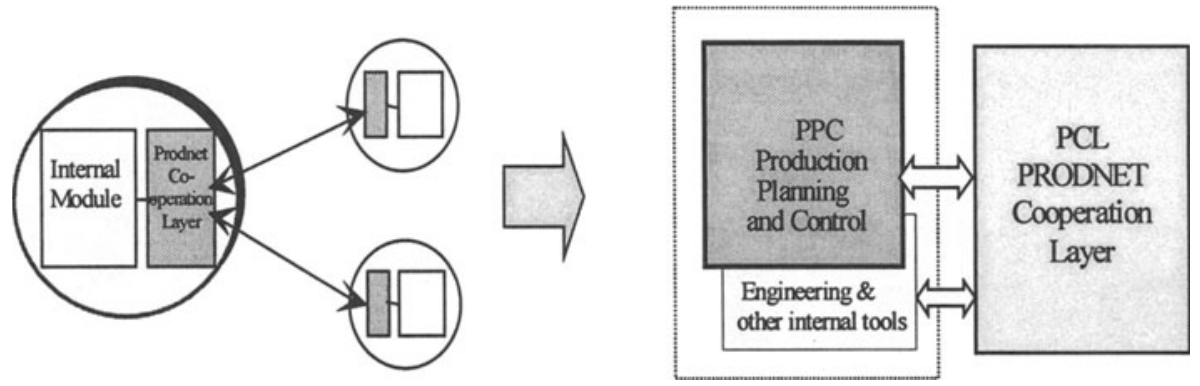

Figure 1 - The PPC in the PRODNET Architecture

In order to investigate the necessary extensions and re-engineering in a typical PPC system, the GEV system from the CSIN partner was used as a test-bed (Figure 2). This system runs on an Oracle database SQL Server and is implemented on a PC / C++ / Windows-NT platform.

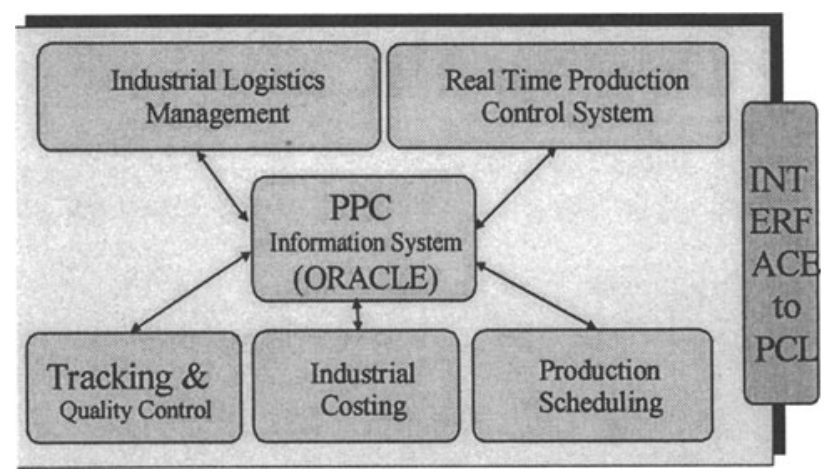

Figure 2 - The architecture of the PPC from CSIN 
This PPC is particularly focused on the needs and specific operating environment of Small and Medium size Enterprises (SME). The main extensions and reengineering applied to this PPC system are illustrated in the gray area in the figure 3 and include the following aspects:

- Support for business and product-related information exchange based on standards (EDIFACT and STEP);

- Support of a reactive behavior regarding events generated by the VE network (via the PCL);

- Support for information requests coming from the VE partners regarding the orders and production status;

- Support for management of incomplete and imprecise orders;

- Support for quality-related information exchange and tracking.

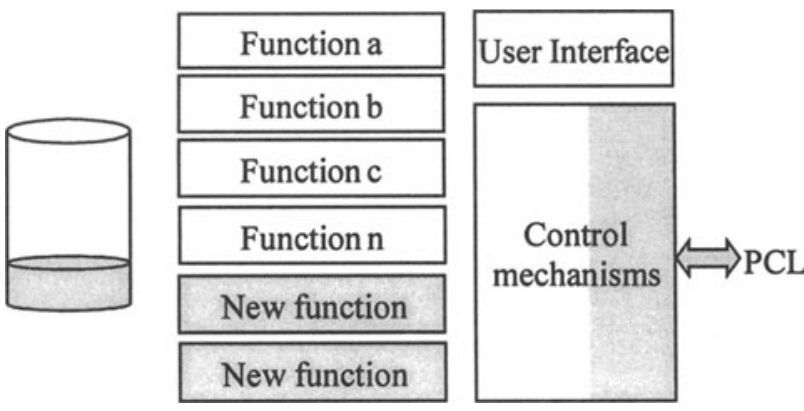

Figure 3 - Areas of re-engineering / extension to the legacy PPC

\section{PPC AND THE INFORMATION EXCHANGE}

\section{Business information exchange}

Orders-related information is the basis of the commercial information exchanged between partners in a VE, since the relationships between client and supplier are mainly based on orders or supply contracts. Orders-related information includes the order data set itself but also quality information about the ordered product. Depending on the case, product models and catalog of products may be exchanged as additional information to an order.

PRODNET II provides EDI-related services, based on the UN/EDIFACT standard promoted by the United Nations, to support the exchange of commercial information. The PPC system is the component in the enterprise that embeds the knowledge to manage the orders-related messages. The consequences for the PPC of the adoption of EDIFACT were twofold:

1) It was necessary to adapt some of the internal data models in order to be compliant with the (chosen subset of) the EDIFACT reference model;

2) The "control structure" of the PPC system had to be extended in order to accept information coming from the VE network via PCL. 
Regarding the first aspect, an alternative could be to keep the internal models of the PPC and to develop a mapping layer to support the data formats compatibility. However, as the PPC producer was a partner in the project, it was decided to follow the first approach, which is also in the direction of the market tendency as other PPC, vendors have been making an effort to adopt EDIFACT.

In terms of the control structure, it shall be noted that most legacy systems were not developed to receive inputs coming from the network. In this way, the typical input to a PPC system is made by human operators via specific window-interfaces. The capability to receive data such as orders and other business data or information requests from the network implied extending the control mechanisms to receive notification or information request events from the PCL. The main advantage of this approach is to allow an "automatic" integration /consumption of orders-related data in the MRP component of the PPC system. Another advantage is the support to distributed business process management. The VE coordinator may ask the VE partners to provide information on the status of a given order / business process.

In order to illustrate the followed implementation approach, let us consider an example of sending an order from company A to company B. The following steps are involved:

Company A:

- The order is prepared in the PPC

- The order is temporarily stored in the PCL

- PCL is notified to start an appropriate workflow plan to send the order out. This might involve several internal steps in the PCL such as format the order according to EDIFACT, optionally encrypt the information, send it to company $\mathrm{B}$, delete the information from PCL storage.

- PPC is notified about the result of the operation.

Company B:

- PCL receives message, stores it internally and activates an EDI parser to decode it.

- The decoded order is sent to the PPC. In alternative, depending on the local workflow plans, the human operator could be notified and asked to decide if the order should be sent to the PPC or not.

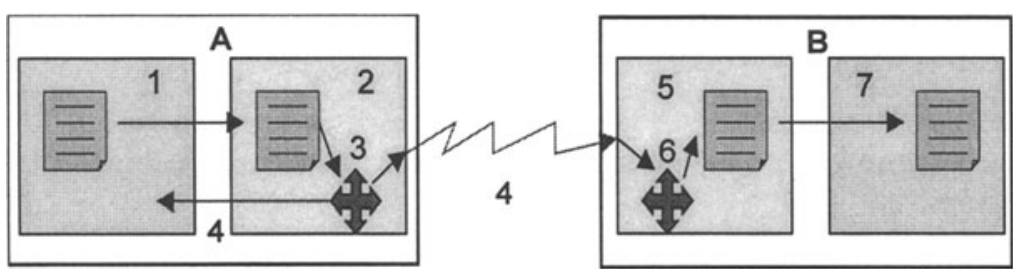

Figure 4 - Sending an order example

\section{Technical product data exchange}

Some orders may carry product-related technical information. This can occur either when the VE goal is related to PDM (Product Data Management) or when a client 
wants to order a new product or component and a product model is necessary to accurately define the requirements. A negotiation process related to a product design can be carried out supported by the interchange of these electronic product models. The STEP standard provides a normalized way to support the exchange of product models. It allows the expression, in a uniform and complete way, of the whole information required for a product during its life cycle. Thus, two companies working in the same industrial sector can model and exchange their product data according to an Application Protocol (AP) defined for that sector.

In the PRODNET II implementation, STEP data is integrated with EDIFACT via the CONDRA message. Once PCL decodes a CONDRA message, resorting to its EDI and STEP modules, the technical product data is normally forwarded to an internal PDM (Product Data Management) or CAD application.

The PPC system can use some of these technical data, namely the information about the product structure (BOM: Bill of Materials). Therefore a new functionality was added to the legacy PPC system in order to import such data. Two input ports were developed: import BOM from a STEP model, and import BOM from a SOLIDWORKS model.

Specific software modules, namely the INTRAVISION from the ProSTEP partner, or the SOLIDWORKS viewer can be activated from the PPC system in order to give the human operator a graphical representation of the product.

\section{ORDERS AND PRODUCTION STATUS FOLLOW UP}

The electronic exchange of orders is a basic functionality to be provided by a VE supporting platform. Besides the exchange of the order itself, it is necessary to support its follow up in order to cope with, for example, delays in the order processing, temporary incapacity of a supplier, the need to re-adjust delivery times, and so on. A client company might even want to know details about the manufacturing status of ordered products or components in order to prevent any difficulties for itself. Orders management is, therefore, one of the most important functionalities to be supported in a VE environment.

In the PRODNET II approach there are two kinds of orders to be handled: client orders and production orders. Client orders, comprising supply and purchase orders, are the ones related to EDI-based business transactions and are fundamentally managed by the PPC and the PCL module. Production orders refer to the business processes effectively contracted by the VE coordinator from its suppliers for the VE goal accomplishment.

\section{Client Orders}

In the PRODNET II reference architecture it is assumed that efficient orders management requires that the company have a PPC system that supports the internal orders management. Without a PPC system it would be unfeasible to manage all required information just at the PCL level. Therefore, a basic requirement in order to accomplish the orders management is a strong interaction between the PCL and the PPC system. This implies some reengineering of the legacy PPC system in order to 
make it reactive to requests coming from the network and to make it able to "understand" the chosen EDI format.

Figure 5 shows the adopted approach for interactions between the PPC and the PCL.

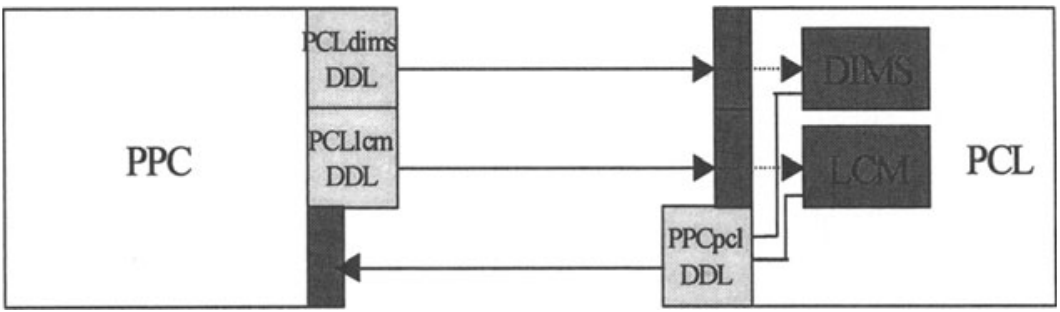

Figure 5 - Interactions between PPC and PCL

These interactions involve the following cases:

- PPC-LCM: when the Local Coordination Module (LCM) of PCL needs to activate a service offered by the PPC (e.g. start order reception) or when PPC wants to activate a service offered by PCL (e.g. send an order to a VE partner).

- PPC-DIMS: The Distributed Information Management Module (DIMS) of PCL offers temporary storage for data arriving at PCL that will be read by the PPC (after some local processing in PCL), data that PPC wants to send out (information items necessary for building an EDIFACT order message), or data that the PPC wants to share with other companies.

The PPC makes its services available via the Dynamic Linking Library (DLL) PPCpcl. Such services can be requested via the function PPC_ServiceRequest. Similarly, the PPC can request a service from LCM or DIMS via the corresponding LCM_ServiceRequest or DIMS_ServiceRequest functions. The corresponding module, via a ServiceAnswer function will inform the conclusion of a requested service.

For illustration purposes, let us consider the interactions involved in sending out an order:

1. The PPC stores the order data in DIMS via DIMS_ServiceRequest.

2. DIMS informs PPC of the result of this request via PPC_ServiceAnswer.

3. If the answer received by PPC is PCL_SUCCESS then it calls LCM to start the appropriate workflow plan to format and send the order out; calling LCM_ServiceRequest with the adequate parameters does this.

4. PCL processes this request executing the indicated workflow plan (one of the core services offered by PCL).

5. When the process is finished, LCM notifies PPC via the PPC_ServiceAnswer function.

Inside the PPC, orders can evolve through several stages along the time, according to their status of accomplishment. Thus, it shall be possible to query the PPC in order to know what is exactly happening to every single client order at any moment.

In PRODNET II an order life cycle, as illustrated in the figure 6, includes the following main states: 
- Received : a new order is received;

- Confirmed: the order is confirmed, the production phase can start;

- Partially shipped: the product(s) required by the order is (are) partially shipped to the client, while the remaining part is still in production;

- Production: the order is in production;

- Fulfilled: the order execution is completed;

- Cancelled: the order is cancelled.

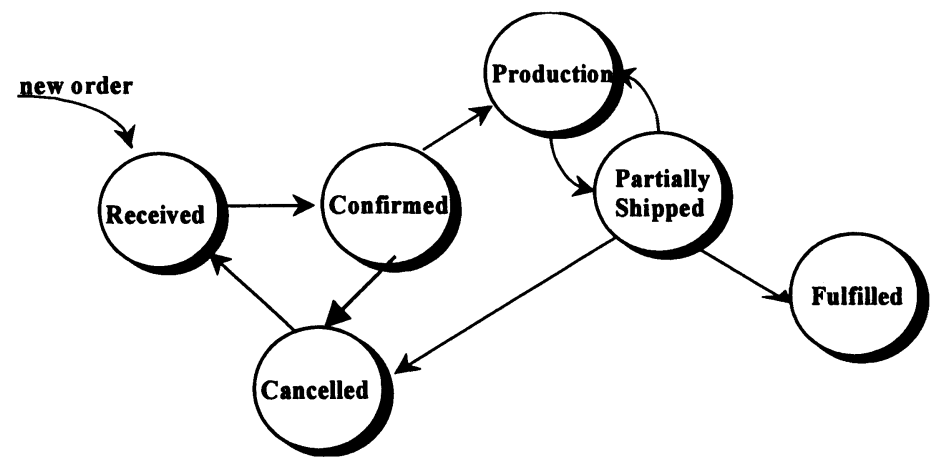

Figure 6-Order's life cycle diagram

\section{Production orders}

In a VE environment business processes are executed in a distributed way, taking into account the contributions of the various VE members. A VE coordinator has to supervise this execution, which implies interacting with the various VE members and therefore requesting information from them their part of the business process (order status, planned and real starting and ending dates, manufactured quantity, etc.). The PPC system of each VE member is the main information source to satisfy these requests.

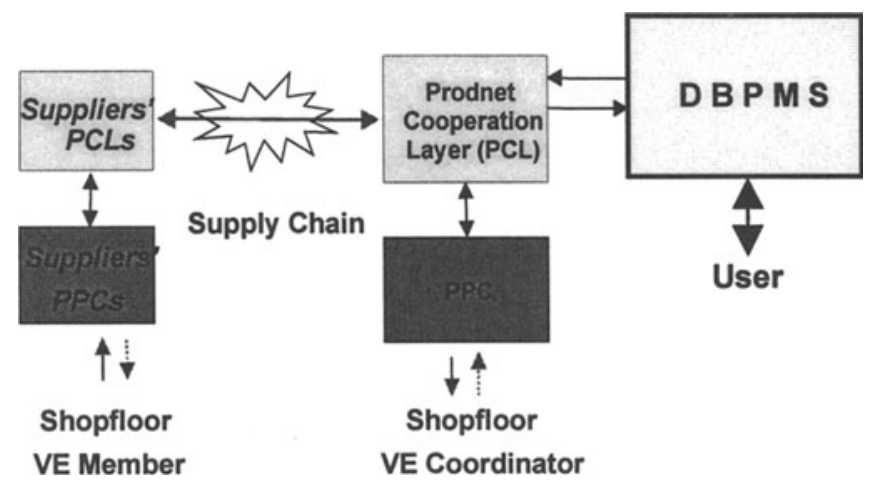

Figure 7 - Support to distributed business process management

In order to support the Distributed Business Process Management System (DBPMS) of the PRODNET infrastructure, a set of specific functions was 
implemented in the PPC (Figure 7). When requested, via a specific parameter in the PPC_ServiceRequest function, the corresponding specialized function will store in DIMS the requested information. DIMS will make the information available to the DBPMS in order to allow intelligent monitoring and decision-making support in case of deviations in the VE schedule. When DBPMS requires information from other enterprises the process is made transparent via the federated query processing implemented by the Distributed Information Management System (Afsarmanesh et al., 1999).

\section{INCOMPLETE AND IMPRECISE ORDERS MANAGEMENT}

\section{The concept}

PRODNET II considers three types of client orders: Regular, Incomplete and Imprecise. There is no information vagueness (incomplete or imprecise data) in a regular order, which means all information related to that order is known. On the other hand, an incomplete order has at least one piece of data missing, whilst an imprecise order has at least one piece of data specified in a vague way. These two types of orders require a special handling process as the data required to fulfilling the order would arrive incrementally. In spite of the incompleteness or vagueness of information, it is often possible for a company to start the production based on the available information as well as to propagate that vagueness to its suppliers according to the MRP/BOM information. This functionality was included as an extension to the legacy PPC system and represents an innovative concept.

Let us consider, for example, an order for a car model $\mathrm{M}$, for which it is possible to specify particular features (optional characteristics), like the type of engine, the color, etc. An incomplete order does not include all required product details. An order could, for instance, simply specify a car model M. If an order like this is received, then it is possible to start the production of the chassis, the doors, etc., independently of the missing information about complementary attributes. New "orders" (or messages) are supposed to arrive in future to complement the missing attributes in the "original" order (these "orders" are not really new orders but additions to the original one). Thus they have to be associated to the original one (logically merged). This process continues until all details about the required product have been specified.

An imprecise order is an order in which the value of some attribute although not missing, is specified in a vague way. For instance, in the car example, the attribute color, in the initial specification, could say "dark color" or "one of blue, black, green". Only later on this attribute would get a specific (precise) value. Another example of vagueness could be on the quantity. An order could specify a tentative amount of between 100 and 120 units (to be confirmed later).

Another situation occurs when a final product is essentially composed of one component or raw material. In this case, it is possible to overcome the imprecision / incompleteness regarding the product by forcing a commitment to a precise quantity of that component. This type of contract is a common practice in the garment industry. For instance, when an order for suits is not precise in terms of quantities and other model-related details, it is common to establish a contract in which the 
client commits himself to use / consume a given amount of the main component (a particular kind of fabric) independently of the product details to be specified later. PRODNET II considers both types, as an important requirement for the orders management.

\section{Impacts on the PPC functionalities}

The management of incomplete and imprecise orders has impacts on various other functions of the PPC, namely: Orders management module, Materials Requirements Planning module, Stocks management module, and Scheduling module.

Two perspectives of vagueness are considered in the implemented system:

1. Vagueness about the particular characteristics of the product (model, size, finishing properties, etc.)

2. Vagueness about other attributes such as delivery data, level of order confirmation, quantity, etc.

Regarding the first perspective, the followed approach uses the part / product code to specify those characteristics, as illustrated in Figure 8.

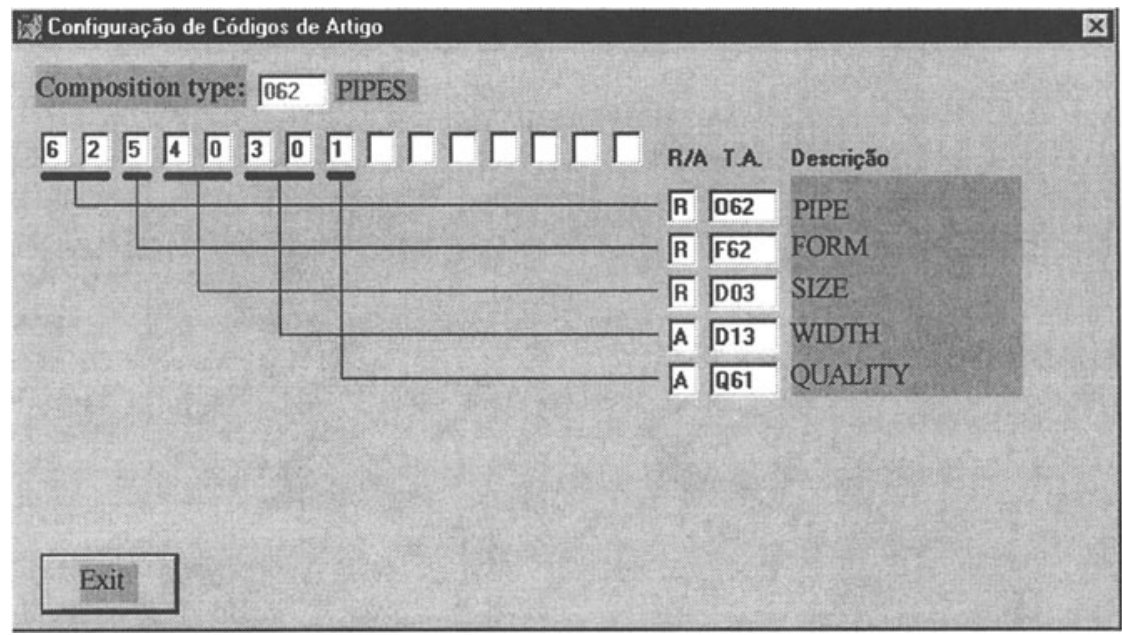

Figure 8-Particular characteristics of a part are represented in its code

Figure 9.a shows a part and its component before the type of finishing properties was specified and Figure 9.b shows the same product after the finishing requirements were received for the same order. 


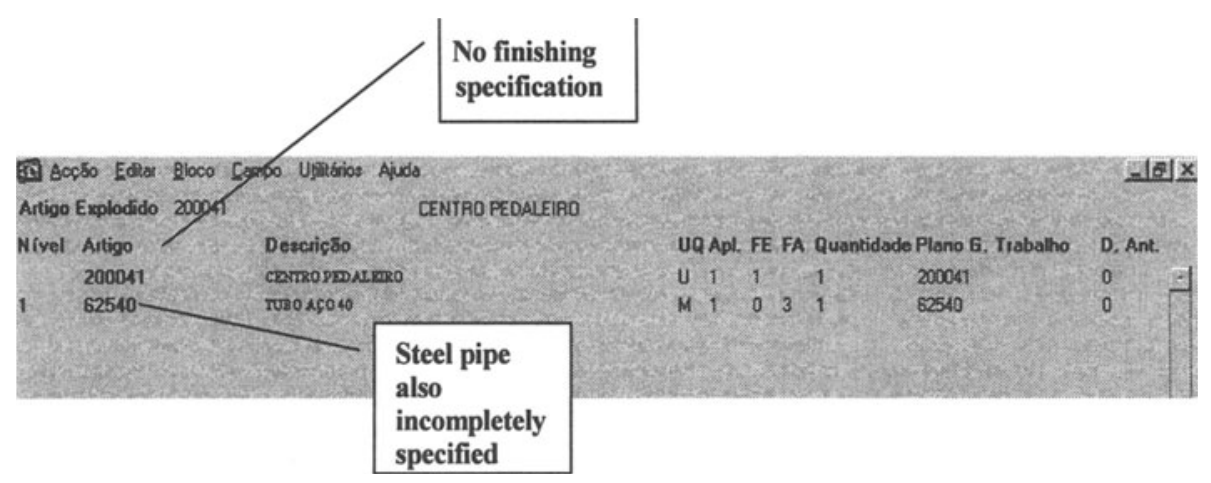

Figure 9.a - Example of a vague product specification

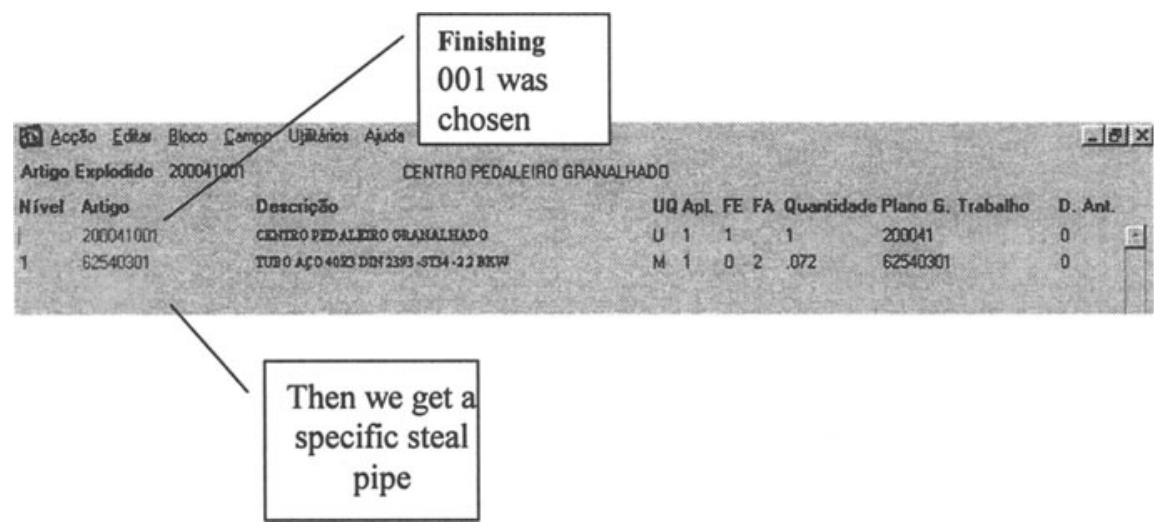

Figure 9.b - Product specification after new information is received

Regarding the second perspective, specific fields in the order may be updated along the time, such as quantity, dates, etc.

The treatment of forecast orders is done resorting to the basic mechanisms developed to manage imprecise / incomplete orders. A forecast order is not a real order but an expectation, usually based on past statistics. For instance, a forecast order for a given product $\mathbf{P}$ for the geographical region $\mathrm{A}$ can be generated with an estimated quantity and undefined finishing properties and delivery dates. Each time a new firm order for product $\mathrm{P}$ arrives from region $\mathrm{A}$, the forecast order is split in two: one corresponding to the firm order and another (which remains incomplete / imprecise) keeping the difference between the initial estimate and the actual ordered amounts (Figure 10). 


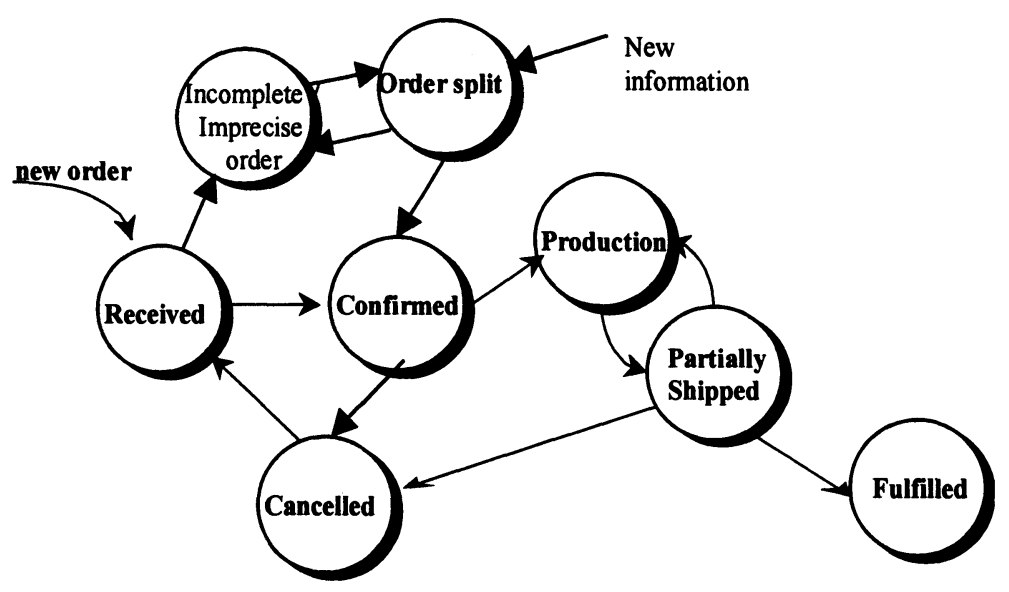

Figure 10 - Extended life cycle for incomplete / imprecise orders

\section{QUALITY-RELATED INFORMATION EXCHANGE}

Another important aspect when "inserting" a company in a VE environment is the proper management of quality related information. In order to allow the definition of liabilities regarding quality in a scenario of distributed manufacturing it is necessary that each node is able to track all components involved in its products. This functionality was also included in the developed system.

The quality management functionality developed in the PPC system includes the following aspects:

- Configuration of general tables to support the quality control procedure. These tables include aspects such as Classes of tests (chemistry, mechanical, component level, system level, etc), Characteristics to be measured / tested (net weight, gross weight, etc), Measurement units, Parts reception codes (normal, controlled by controller, controlled by chief controller, etc), etc. In this definition the EDIFACT recommendations were followed.

- Definition of rules for technical Parts Reception (quality verification process).

- Launching of an automatic control procedure according to the quality verification conditions configured for each supplier / partner. In other words, the system can monitor and enforce the verification process defined for each supplier. This process involves the management of all associated documents and generation of verification commands for the Quality department.

The verification procedure to apply may vary from supplier to supplier. Suppliers are classified according to a number of factors reflecting their past behavior regarding quality aspects and compliance with ISO 9000 . 


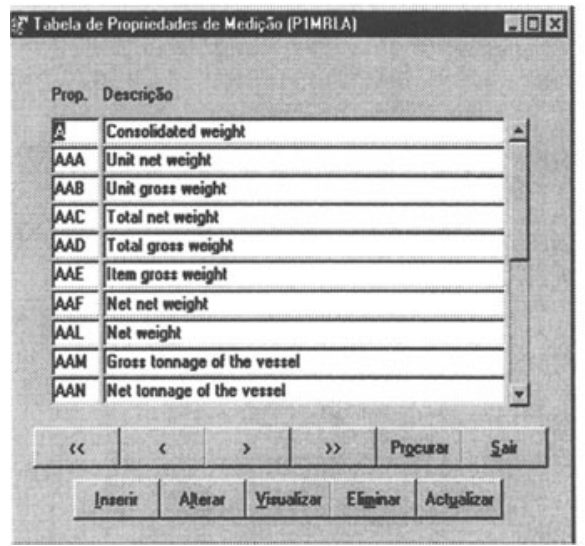

Figure 11.a - Example of specification of characteristics to be measured

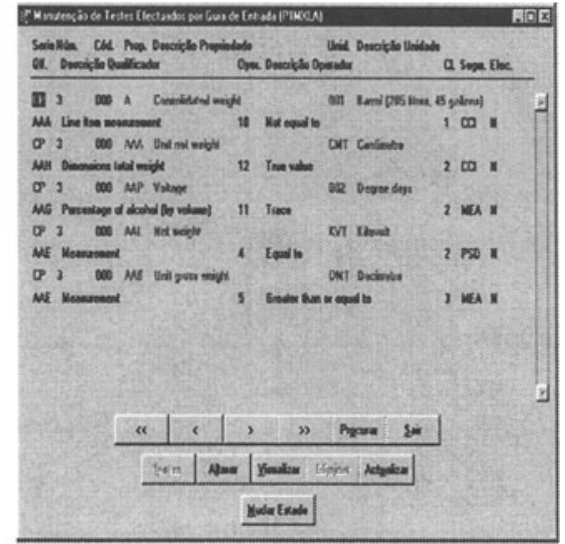

11.b - Example of tests for a part reception

Results of the parts' acceptance procedure, together with a reference to the corresponding suppliers / partners, are kept in a historic database allowing for future trace of the distributed responsibilities involved in each product.

\section{CONCLUSIONS AND FUTURE DIRECTIONS}

A set of extensions and a re-engineering process were developed and applied to a legacy PPC system in order to support its operation in a VE environment. The main developments include:

- Orders and production status follow up, answering requests coming from the network;

- Support to EDIFACT compliant interactions;

- Interface to STEP based BOM;

- Management of incomplete and imprecise orders;

- Quality management taking into account the full supply chain.

From the evaluation and experimental work developed in PRODNET II, it became clear that the functionality of future PPC systems will move away from an internal focus on the company functions and extend into and across the VE network. A similar tendency is observed in the ERP systems (Bond, 1997). This extended perspective will require a complete redesign of the architecture of these systems in order to better take into account rapid business process changes. This approach is also consistent with the recent guidelines being proposed by the Open Applications Group (OAG, 1997).

Other extensions in the PPC are related to the impact its change will cause on the business processes. So far some evaluation studies were carried out in terms of social and organizational implications. These impacts can also have many consequences in the way other enterprise activities and tools should operate.

New emergent concepts have been investigated and can be seen as future trends to take into account in the "new networked generation" of PPCs. Decision-making 
has its complexity and time constraints are highly increased in a VE environment, creating more obstacles for agile and intelligent reactions of the enterprise in the presence of changes and problems in the supply-chain. In this sense, negotiationbased assisted systems represent an advanced concept and technology that can be applied in the scheduling module of the PPCs (Rabelo and Camarinha-Matos, 1998). Working cooperatively, the production resources from different enterprises can virtually and logically be "rented" to another enterprise in case of production overloading. The coordination of such complex and tight networked cooperation can be supported by the multi-agent systems paradigm (Rabelo et al., 1999).

A truly networked PPC should more gradually be integrated with the other systems of the PRODNET platform in order to keep working with update information from the supply-chain. For example, the VE reschedule generated by the DBPMS module when a serious problem takes place in the supply-chain should be (ideally) automatically loaded into the PPC system instead of waiting for the human approval. Although this manual action seems to provide a more "reliable" information to a PPC, it can take too long, as an enterprise will normally be involved in several VEs simultaneously. Thus, more effective working methods should be applied in order to make the decision process and the PPC updating more agile.

So far the PPCs "think" in an isolated way. Working under the integrated logistics management philosophy, the cooperation among the VE members is a must in order to reduce the global waste and hence to maximize the profits and workload. It is a kind of extension strategically important to be added to modern PPCs, providing DRP and DRP-II capabilities (distributed manufacturing requirements planning / distributed manufacturing resources planning).

In the PRODNET II approach, the rights and duties of the VE participants in terms of information access are expressed in the so-called "supervision clauses". Their specification is manually configured, so far, once a VE is created. In the near future this specification could be "automatically" derived, based on the "new generation" of contracts for the VE environment. Therefore, the PPC should be prepared to feed the involved databases with authorized information only.

The developed extensions to a PPC represent a first step in this direction, but there is clearly a need to evolve to a more modular / "fragmented" architecture in which the enterprise functions are organized as a library of services that can be used in a very flexible way according to the dynamic workflow plans defined for each enterprise and each business plan.

\section{Acknowledgments}

This work was funded in part by the European Commission, Esprit program within the PRODNET II project and the Brazilian research council (CNPq).

The authors also thank the valuable contributions from the consortium partners: CSIN (P), ESTEC (P), HERTEN (BR), LICHEN Informatique (F), MIRALAGO (P), ProSTEP (D), UNINOVA (P), University of Amsterdam (NL), Universidade Federal de Santa Catarina (BR), and Universidade Nova de Lisboa (P). 


\section{REFERENCES}

1) Afsarmanesh, H.; Garita, C.; Ugur, Y.; Frenkel, A.; Hertzberger, L.O. - Design of the federated information management architecture for PRODNET, in Infrastructures for Virtual Enterprises, L.M. Camarinha-Matos and H. Afsarmanesh (Eds.), Kluwer Academic Publishers, 1999.

2) Bond, B. - ERP Vision: From enterprise to extended enterprise, Gartner Group report, www.camus.org/Publicat/News/1997FalA.htm

3) Camarinha-Matos, L.M.; Pantoja Lima, C. - A Framework for Cooperation in Virtual Enterprises, Proceedings of DIISM'98 - Design of Information Infrastructures Systems for Manufacturing 1998, Fort Worth, USA, May 98.

4) OAG - Open Applications Integration White Paper, http://www.openapplications.org.

5) Rabelo, R. J.; Camarinha-Matos, L. M.; Afsarmanesh, H.- Multiagent-based Agile Scheduling, International Journal of Robotics and Autonomous Systems, Special issue on Multi-Agent Systems Applications, North-Holland, vol. 27, N. 1-2, April 1999.

6) Rabelo, R. J.; Camarinha-Matos, L. M. - Generic framework for conflict resolution in negotiation-based agile scheduling systems, Proceedings of IMS'98 $-5^{\text {th }}$ IFAC Workshop on Intelligent Manufacturing Systems, Gramado - Brazil, November 1998. 